\title{
N $94-13310$
}

\section{IMPROVEMENTS IN HOVER DISPLAY DYNAMICS FOR A COMBAT HELICOPTER}

\author{
Jeffery A. Schroeder \\ Aerospace Engineer \\ NASA Ames Research Center \\ Moffett Field, California
}

\author{
Michelle M. Eshow \\ Aerospace Engineer \\ Aeroflightdynamics Directorate \\ U.S. Army ATCOM \\ Moffett Field, California
}

\begin{abstract}
This paper describes a piloted simulation conducted on the NASA Ames Vertical Motion Simulator. The objective of the experiment was to investigate the handling qualities benefits attainable using new display law design methods for hover displays. The new display laws provide improved methods to specify the behavior of the display symbol that predicts the vehicle's ground velocity in the horizontal plane; it is the primary symbol that the pilot uses to control aircraft horizontal position. The display law design was applied to the Apache helmet-mounted display format, using the Apache vehicle dynamics to tailor the dynamics of the velocity predictor symbol. The representations of the Apache vehicle used in the display design process and in the simulation were derived from flight data. During the simulation, the new symbol dynamics were seen to improve the pilots' ability to maneuver about hover in poor visual cuing environments. The improvements were manifested in pilot handling qualities ratings and in measured task performance. The paper details the display design techniques, the experiment design and conduct, and the results.
\end{abstract}

\section{NOTATION}

$A_{x} \quad$ acceleration cue longitudinal position, deg (degrees refer to angle subtended at pilot's eye)

$A_{y} \quad$ acceleration cue lateral position, deg Error $_{\text {nor } t h}$ vehicle earth-axis position error northward, $\mathrm{ft}$ Error east vehicle earth-axis position error eastward, $\mathrm{ft}$ $f_{i}(s) \quad$ sensor equalization filter on signal i

$g \quad$ gravity constant, $\mathrm{ft} / \mathrm{sec}^{2}$

$K_{x} \quad$ display longitudinal conversion factor for hover box, deg/ft

Presented at Piloting Vertical Fight Aircraft: A Conference on Flying Qualities and Human Factors, San Francisco, Califomia, 1993. Originally published as an altemate paper of the 48th Annual Forum of the American Helicoper Society, Washingion, D.C., 1992.

\begin{tabular}{|c|c|}
\hline$K_{y}$ & $\begin{array}{l}\text { display lateral conversion factor for hover box, } \\
\text { deg/ft }\end{array}$ \\
\hline$K_{\dot{x}}$ & $\begin{array}{l}\text { display longitudinal conversion factor for } \\
\text { velocity vector, deg/ft/sec }\end{array}$ \\
\hline$K_{\dot{y}}$ & $\begin{array}{l}\text { display lateral conversion factor for velocity } \\
\text { vector, } \mathrm{deg} / \mathrm{ft} / \mathrm{sec}\end{array}$ \\
\hline$L_{\delta_{a}}$ & $\begin{array}{l}\text { vehicle derivative of applied specific rolling } \\
\text { moment due to lateral cyclic, } \mathrm{rad} / \mathrm{sec}^{2} / \mathrm{in} \text {. }\end{array}$ \\
\hline$M_{\delta_{b}}$ & $\begin{array}{l}\text { vehicle derivative of applied specific pitching } \\
\text { moment due to longitudinal cyclic, } \mathrm{rad} / \mathrm{sec}^{2} / \mathrm{in} \text {. }\end{array}$ \\
\hline $\begin{array}{l}P_{x} \\
P_{y}\end{array}$ & $\begin{array}{l}\text { hover box longitudinal position, deg } \\
\text { hover box lateral position, deg }\end{array}$ \\
\hline$p$ & vehicle body-axis roll rate, $\mathrm{rad} / \mathrm{sec}$ \\
\hline$q$ & vehicle body-axis pitch rate, rad/sec \\
\hline & Laplace operator \\
\hline$V_{\text {north }}$ & $\begin{array}{l}\text { northward component of vehicle groundspeed, } \\
\mathrm{ft} / \mathrm{sec}\end{array}$ \\
\hline$V_{\text {east }}$ & $\begin{array}{l}\text { eastward component of vehicle groundspeed, } \\
\mathrm{ft} / \mathrm{sec}\end{array}$ \\
\hline $\begin{array}{l}V_{x} \\
V_{y}\end{array}$ & $\begin{array}{l}\text { velocity vector longitudinal position, deg } \\
\text { velocity vector lateral position, deg }\end{array}$ \\
\hline$x^{9}$ & vehicle longitudinal position, $\mathrm{ft}$ \\
\hline $\begin{array}{l}x_{c m d} \\
\dot{x}\end{array}$ & $\begin{array}{l}\text { commanded vehicle longitudinal position, } \mathrm{ft} \\
\text { longitudinal heading referenced groundspeed, } \\
\mathrm{ft} / \mathrm{sec}\end{array}$ \\
\hline$\dot{x}_{f i l t}$ & filtered longitudinal groundspeed, $\mathrm{ft} / \mathrm{sec}$ \\
\hline$\ddot{x}_{\text {comp }}$ & $\begin{array}{l}\text { complementary filtered longitudinal acceleration, } \\
\mathrm{ft} / \mathrm{sec}^{2}\end{array}$ \\
\hline$\ddot{x}_{f i l}$ & estimated longitudinal acceleration, $\mathrm{ft} / \mathrm{sec}^{2}$ \\
\hline$X_{u}$ & vehicle longitudinal velocity damping, $1 / \mathrm{sec}$ \\
\hline$\dot{y}$ & lateral heading referenced groundspeed, $\mathrm{ft} / \mathrm{sec}$ \\
\hline$\dot{y}_{f i l t}$ & filtered lateral groundspeed, ft/sec \\
\hline$\ddot{y}$ comp & $\begin{array}{l}\text { complementary filtered lateral acceleration, } \\
\mathrm{ft} / \mathrm{sec}^{2}\end{array}$ \\
\hline$\ddot{y}_{f i l}$ & estimated lateral acceleration, $\mathrm{ft} / \mathrm{sec}^{2}$ \\
\hline & $\begin{array}{l}\text { vehicle lateral velocity damping, } 1 / \mathrm{sec} \\
\text { pilot lateral cyclic control position, in. }\end{array}$ \\
\hline
\end{tabular}


$\delta_{b} \quad$ pilot longitudinal cyclic control position, in.

$\zeta \quad$ damping ratio

$\theta \quad$ vehicle Euler pitch angle, rad

$\dot{\theta} \quad$ vehicle Euler pitch rate, $\mathrm{rad} / \mathrm{sec}$

$\phi \quad$ vehicle Euler roll angle, rad

$\dot{\phi} \quad$ vehicle Euler roll rate, $\mathrm{rad} / \mathrm{sec}$

$\psi \quad$ vehicle heading angle, rad

$\omega \quad$ natural frequency, $\mathrm{rad} / \mathrm{sec}$

\section{INTRODUCTION}

A significant effort at Ames Research Center has aimed at developing and flight testing display law design methods for the hover flight regime. The flight experiment of Ref. 1 documented the influence of display dynamics on handling qualities for near-hover maneuvering; the Ref. 2 flight experiment examined the relative merits of two pilot-oriented design goals for the display dynamic response. Both experiments employed a cockpit panel-mounted representation of the AH-64 Pilot Night Vision System (PNVS) symbology (Ref. 3), which is shown in Figure 1. The flight experiment of Ref. 4, following many years of simulation research, examined control and display requirements for VTOL translation, hover, and landing, using an Ames-designed symbology format.

The common theme for all the experiments was the use of a velocity predictor symbol (called the acceleration cue in Figure 1). The emphasis of the research was placed on the specification of that symbol's dynamics. When used with the hover position symbol and the velocity vector, the acceleration cue is the pilot's primary controlled element for regulation of vehicle horizontal position. Although the acceleration cue predicts future horizontal velocities, it is used primarily in combination with another symbol that indicates a desired vehicle horizontal position, to control vehicle horizontal position. For helicopters with angular rate stabilization only, the resulting aircraft position dynamics are difficult to control, as there are approximately three integrations from pilot input to aircraft position response. This separation of the pilot from the vehicle state of interest presents a handling qualities challenge to the display designer. As will be described subsequenty, the acceleration cue response to pilot control input must be designed considering the vehicle dynamics and the task requirements to maximize handling qualities and mission effectiveness.

The lessons leamed from the three fight experiments provided the foundation for the flight investigation of Ref. 5 , whose objectives were 1) to design new display laws tailored specifically to the Apache vehicle dynamics and 2) to compare the resulting handling qualities with those of the existing Apache display laws. While the first objective was achieved, the second was not because the documented representation of the existing Apache display laws used in the flight comparison was not correct. The correct display laws were obtained subsequently, and potential improvements were then shown analytically.
Since that experiment, as will be described, flight data documenting the Apache vehicle response characteristics were obtained that permitted the identification of highquality design and simulation models. The nature of the identified vehicle response necessitated an extension of the display law design methods described in Ref. 2 and Ref. 4. Thus, the motivation for the simulation experiment described here was to examine the potential benefits of the extended design methods using an improved representation of the Apache vehicle and of its baseline display responses. The following sections detail the display law design methods, the simulation design and conduct, and the results.

\section{DISPLAY LAW DESIGNS}

The term "display laws" refers to the equations and scaling that determine the position of the central symbology, namely the acceleration cue, velocity vector, and hover position box (Figure 1). During hover maneuvering using primarily the symbology, the acceleration cue becomes the pilot's primary controlled element. To achieve a hover over the position box, he moves his stick to place the cue on the box, and he maintains it there as the box converges to the display center. The pilot workload to maintain the cue on the box, and the nature of the resulting vehicle trajectory, are the two issues that most impact the design of the acceleration cue dynamics.

These considerations are illustrated in Figure 2, which presents a block diagram of the pilot-vehicle-display system for the case where the pilot is attempting to zero the longitudinal displayed error between the hover box and acceleration cue. The ease of controlling the acceleration cue's position on the display is determined by the transfer function $A_{x} / \delta_{b}$, which in turn is determined by the cue's response to each of the aircraft states that drive it.

Given any particular set of dynamics for the cue response to control, the trajectory that the aircraft follows while the pilot maintains the cue on the hover box is determined by the closed loop response $x / x_{c m d}$. This response must be tailored so that the trajectory is well-damped, with a bandwidth, or "aggressiveness," appropriate for the aircraft mission.

There is a tradeoff between the cue controllability, which affects the pilot workload, and the aircraft position response. In one extreme, the easiest cue to control would be one driven only by pilot control position; however, this would result in poor hovering performance. This problem has been referred to as poor "face validity" (Ref. 6). In the other extreme, the cue position could be driven to show the pilot control inputs required for a quick, well-behaved trajectory, probably resulting in complex control motions and high workload. Finally, the tradeoffs become more critical as the level of vehicle augmentation decreases, since stability margins deteriorate quickly. 


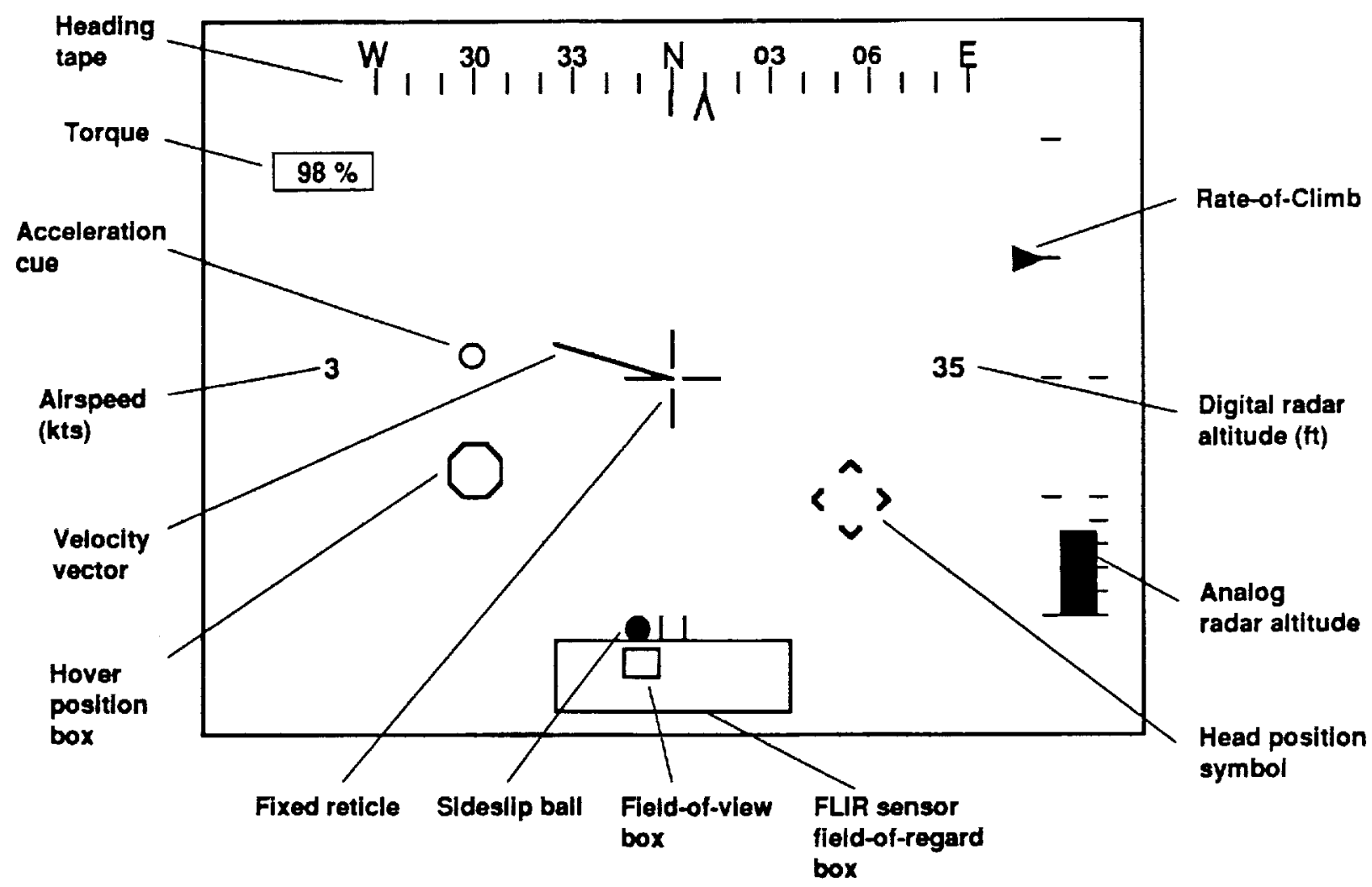

Fig. 1 AH-64 Pilot Night Vision System symbology.

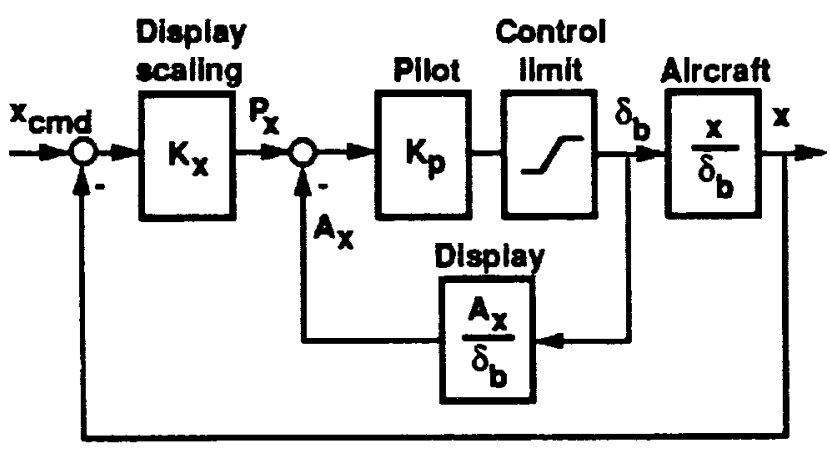

Fig. 2 Pilot-vehicle-display block diagram.

With these guidelines in mind, three methodologies for specifying display laws were examined for the experiment. After brief discussions of the vehicle dynamics model used for the display designs and of the baseline production display laws, a description of each design method is presented. Finally, all the display laws are compared analytically.

\section{Vehicle Design Model}

To support the display law design, a mathematical model was needed of the AH-64 Apache (Figure 3) with its Digital Automatic Stabilization Equipment (DASE) on. Parameter identification techniques described in Ref. 7 were used to identify from flight data a low-order model for the
DASE-on vehicle near hover. The flight data were part of a larger AH-64 database generated by the Amny at the Airworthiness Qualification Test Directorate (AQTD); the flight tests are described in Ref. 8.

The DASE-on design model has decoupled transfer functions with associated equivalent time delays for the longitudinal and lateral responses to pilot input. These were the only responses required for the display design. The following models were identified from flight data that exhibited

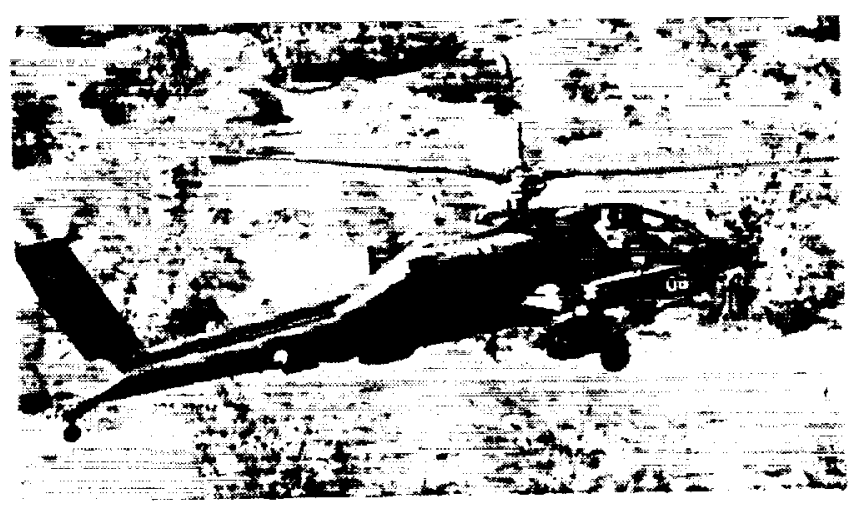

Fig. 3 AH-64 Apache. 
excellent coherence in the frequency range of interest $(0.2$ to $10 \mathrm{rad} / \mathrm{sec}$ ):

$$
\begin{aligned}
& \frac{q}{\delta_{b}}(s)=\frac{-2.49(s+0.262)}{(s+0.399)[0.805 ; 3.46]} e^{-0.103 s} \\
& \frac{p}{\delta_{a}}(s)=\frac{6.32}{[0.582 ; 4.29]} e^{-0.0425 s}
\end{aligned}
$$

where the shorthand notation indicates the second order system $[\zeta ; \omega]=s^{2}+2 \zeta \omega s+\omega^{2}$. Note that these high-order rate responses approximate, over the fitted frequency range, the combined dynamic effects of the unaugmented vehicle and its limited-authority augmentation system. Previously, two of the display design methods had been applied to only first-order rate responses; those methods had to be extended to accommodate these high-order identified responses.

\section{Production Display Laws}

The PNVS display mode of interest for this study is the Bob-Up mode, which includes the velocity vector, acceleration cue, and hover box symbols. The symbol deflection definitions are shown in Figure 4. Based on unpublished documentation provided by the manufacturer and by the Army's program management office, the equations governing the movement of each symbol are next described.

\section{Hover Position Box}

In the current production version of the PNVS software, the hover box is an octagon drawn and scaled to have an edge-to-edge width of $8 \mathrm{ft}$. It is driven relative to the fixed reticle by the heading-referenced, Earth-axis position error to a pilot-selected point:

$$
\begin{aligned}
& P_{x}=K_{x}\left(\text { Error }_{\text {north }} \cos \psi+\text { Error }_{\text {east }} \sin \psi\right) \\
& P_{y}=K_{y}\left(- \text { Error }_{\text {north }} \sin \psi+\text { Error }_{\text {east }} \cos \psi\right)
\end{aligned}
$$

Here, the errors equal the desired position minus the current position, and the desired position is the one existing

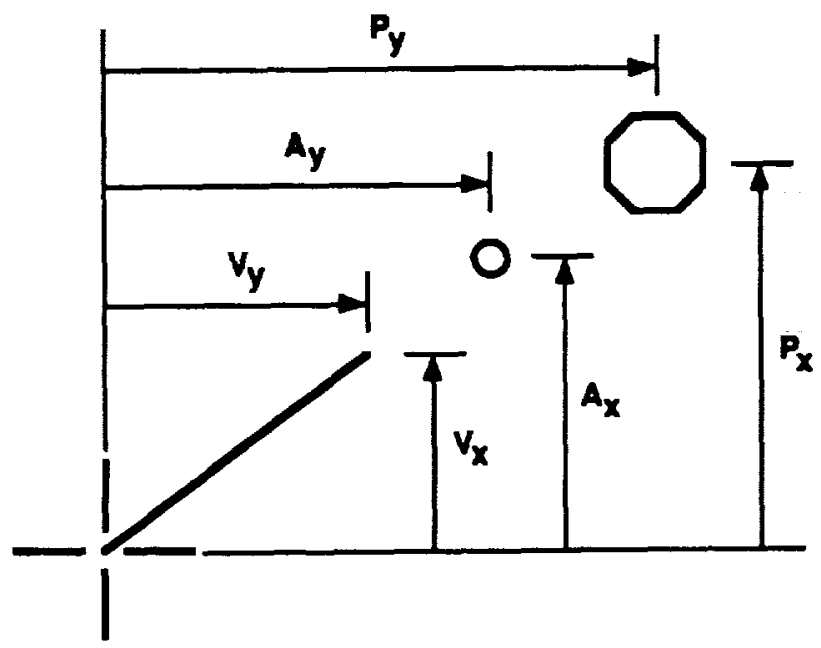

Fig. 4 Definitions of central symbology deflections. when the Bob-Up mode was selected. The hover box moves opposite to the aircraft motion to show the relative location of the desired position. To re-initialize the box to the current vehicle position, centered on the fixed reticle, the pilot desejects then reselects the Bob-Up mode. The scale factors $K_{x}$ and $K_{y}$ are required to convert feet to display displacement, such that full-scale deflection of the center of the box is $\pm 44 \mathrm{ft}$. The full-scale deflection point is such that the outer edge of the box is just below the heading tape. The values of $K_{x}$ and $K_{y}$ were $0.241 \mathrm{deg} / \mathrm{ft}$, where the degrees refer to the angle of display displacement subtended, on the PNVS monacle, at the pilot's eye.

\section{Velocity Vector}

The velocity vector tip location relative to the fixed reticle is calculated as follows:

$$
\begin{gathered}
\dot{x}=V_{\text {north }} \cos \psi+V_{\text {east }} \sin \psi \\
\dot{y}=-V_{\text {north }} \sin \psi+V_{\text {east }} \cos \psi \\
\dot{x}_{\text {filt }}(s)=\frac{1}{(s+1)} \dot{x}(s) \\
\dot{y}_{f i l t}(s)=\frac{1}{(s+1)} \dot{y}(s) \\
V_{x}=K_{\dot{x}} \dot{x}_{f i l t} \\
V_{y}=K_{\dot{y}} \dot{y}_{\text {filt }}
\end{gathered}
$$

Where $K_{\dot{x}}$ and $K_{\dot{y}}$ are again scale factors to convert $\mathrm{ft} / \mathrm{sec}$ to degrees of display displacement They have the value of $1.03 \mathrm{deg} / \mathrm{f} / \mathrm{sec}$ so that the full scale deflection of the vector represents $12.0 \mathrm{ft} / \mathrm{sec}(7.13 \mathrm{knots})$. The velocity vector's full-scale deflection point on the display is $15 \%$ beyond that of the hover box, or midway into the heading tape.

\section{Acceleration Cue}

The acceleration cue center relative to the fixed reticle is calculated as follows:

$$
\begin{aligned}
& \ddot{x}_{f i l t}(s)=\frac{s}{s^{2}+2 s+1} \dot{x}(s)-\frac{32.2(s+2)}{s^{2}+2 s+1} \dot{\theta}(s) \\
& \ddot{y}_{f i l t}(s)=\frac{s}{s^{2}+2 s+1} \dot{y}(s)+\frac{32.2(s+2)}{s^{2}+2 s+1} \dot{\phi}(s) \\
& A_{x}=K_{\dot{x}}\left(\dot{x}_{f i l t}+1.507 \ddot{x}_{f i l t}-3.013 \dot{\theta}\right) \\
& A_{y}=K_{\dot{y}}\left(\dot{y}_{f i l t}+1.507 \ddot{y}_{f i l t}+3.013 \dot{\phi}\right)
\end{aligned}
$$

Thus the acceleration cue is driven relative to the tip of the velocity vector with an estimate of linear acceleration plus some lead compensation generated by the attitude rate terms.

The three new display design methods applied to the PNVS will next be described. It should be noted that for these new display laws, the display scalings of the three symbols remained invariant and equal to those of the production laws to preserve their operational significance and to provide a consistent basis of comparison among all the laws. 


\section{Modified Production Display Laws}

The first display law design method did not fully apply the techniques described in the introduction. Rather, it consisted of simply adjusting the gains on the acceleration and attitude rate terms in the production cue equations and the time constants of the velocity vector filter. The motivation for this design was to investigate whether simple changes in the existing equations, requiring no additional sensor information, would favorably impact handling qualities on $\mathrm{AH}$ 64's in the current fleet. The adjustments were made empirically based on a goal of improving the vehicle position trajectory response when the pilot is adopting the guidance strategy of placing the cue on the position box during the capture.

The transfer function of the controlled element, $A_{x}(s) / \delta_{b}(s)$, that results for the production display laws has an underdamped complex pair of zeros in its numerator (at $-0.48 \pm \mathrm{j} 0.66 \mathrm{rad} / \mathrm{sec}$ ). These underdamped zeros result from the interaction of the display feedbacks with the heavily filtered groundspeed signal. If the velocity filter breakpoint is moved from $1 \mathrm{rad} / \mathrm{sec}$ to $10 \mathrm{rad} / \mathrm{sec}$, the underdamped complex zeros are eliminated. This modification to the sensor filtering alone would likely result in increased cue noise in flight. So in combination with the above filtering change, the gains on high-frequency inputs (accelerations and attitude rates) were lowered. The lowering of these gains was accomplished while trying to achieve vehicle-display dynamics having an integrator-like response to pilot input in the crossover frequency range (Ref. 9). This design was developed during the simulation, and the authors recognize that depending on sensor signal quality in the AH-64, increased gains could improve this cue's response. The final equations for the modified production design were as follows:

$$
\begin{gathered}
\dot{x}_{f i l t}(s)=\frac{1}{(0.1 s+1)} \dot{x}(s) \\
\dot{y}_{f i l t}(s)=\frac{1}{(0.1 s+1)} \dot{y}(s) \\
V_{x}=K_{\dot{x}} \dot{x}_{f i l t} \\
V_{y}=K_{\dot{y}} \dot{y}_{f i l t} \\
A_{x}=K_{\dot{x}}\left(\dot{x}_{f i l t}+1.290 \ddot{x}_{f i l t}-0.286 \dot{\theta}\right) \\
A_{y}=K_{\dot{y}}\left(\dot{y}_{f i l t}+0.800 \ddot{y}_{f i l t}+0.160 \dot{\phi}\right)
\end{gathered}
$$

with $\ddot{x}_{f i l t}$ and $\ddot{y}_{f i l t}$ defined in eqn. 11 and eqn. 12 . Display Laws Based on Workload Design

The second design employed the philosophy developed in Ref. 2 with an extension of that methodology to treat the identified AH-64 aircraft dynamics. Entitled the "workload" design, this method seeks to reduce pilot workload by providing high-frequency proportional, or gain-like, response of the acceleration cue to pilot input while also assuring desirable trajectory response. The handling qualities benefits of the gain-like response goal were established in the flight experiment of Ref. 2, which compared gain-like responses with integrator-like responses for hover maneuvering using the same display format.

In this method, a display law is specified for the cue in terms of a sum of compensated aircraft states and controls. The aircraft dynamics are then considered in order to define a desirable and achievable cue response to pilot control. This desired transfer function is next adjusted if necessary to achieve acceptable trajectory response. Then, the sensor compensation is determined that provides the desired cue response. The details of this approach are now described for the longitudinal and lateral axes.

\section{Longitudinal Axis Design}

The general display law for this method, as extended for this application, is:

$A_{x}(s)=f_{\dot{x}}(s) \dot{x}(s)+f_{\theta}(s) \theta(s)+f_{q}(s) q(s)+f_{\delta_{b}}(s) \delta_{b}(s)$

Where the $f_{i}$ 's represent the sensor signal compensation required to provide the desired cue response. Dividing by $\delta_{b}$ yields:

$$
\begin{aligned}
\frac{A_{x}}{\delta_{b}}(s) & =f_{\dot{x}}(s) \frac{\dot{x}}{\delta_{b}}(s)+f_{\theta}(s) \frac{\theta}{\delta_{b}}(s) \\
& +f_{q}(s) \frac{q}{\delta_{b}}(s)+f_{\delta_{b}}(s)
\end{aligned}
$$

For the desired gain-like cue response to pilot input above some frequency, this transfer function's numerator and denominator must be of equal order. The objective is to determine the order and parameter values for each filter to yield this gain-like cue response. The choices are also constrained by the requirement to provide good trajectory response dynamics. The relationship between the two can be seen by referring to Figure 2, where for high values of pilot gain, $K_{p}$, the open-loop position transfer function may be approximated by:

$$
\frac{x}{x_{c m d}-x}(s) \approx K_{x} \frac{x / \delta_{b}}{A_{x} / \delta_{b}}
$$

Thus, for fixed display position and velocity scalings and vehicle response, tailoring the cue response is the only means of assuring an acceptable closed-loop position response. The cue transfer function can be used, for example, to cancel unwanted dynamics in the vehicle position response to control input. Of course, this must be accomplished while still maintaining good cue controllability.

Next recall that the aircraft longitudinal response has the form (neglecting the transport delay):

$$
\frac{\theta}{\delta_{b}}(s)=\frac{M_{\delta_{b}}(s+a)}{s(s+b)[\zeta ; \omega]}
$$


Substituting the aircraft responses into eqn. 22 with the approximations:

$$
\begin{gathered}
\frac{\dot{x}}{\theta}(s)=\frac{-g}{s-X_{u}} \\
q=\dot{\theta}
\end{gathered}
$$

yields

$$
\frac{A_{x}}{\delta_{b}}(s)=\left[\text { terms in }\left(f_{i}\right)\right] \frac{-g M_{\delta_{b}}(s+a)}{s(s+b)\left(s-X_{u}\right)[\zeta ; \omega]}
$$

This relation is simply the unaugmented vehicle velocity response with added zeros (in the terms in $f_{i}$ ) that can be used to provide lead to the cue position dynamics.

For the overall transfer function to be proper, the transfer function in the brackets must have an excess of four zeros. In addition, it is desirable to cancel the attitude response's lead-lag pair from the trajectory response, to eliminate position overshoot. For these reasons, the following form is chosen for the cue response transfer function:

$$
\frac{A_{x}}{\delta_{b}}(s)=\frac{K_{\delta_{b}}\left(s+z_{1}\right)\left(s+z_{2}\right)(s+a)[\zeta ; \omega]}{s(s+b)\left(s-X_{u}\right)[\zeta ; \omega]}
$$

where $K_{\delta_{b}}$ is a total gain that represents the high frequency cue sensitivity to control input. Note that two zeros are chosen to cancel the complex poles from the cue response, in order to simplify it. However, this means that they will be present in the trajectory response. This choice of zeros may not be appropriate for very poorly damped vehicles and should therefore be considered for each case. The placement of the zeros $z_{1}$ and $z_{2}$ determines the frequency at which the cue response becomes gain-like.

The numerator of eqn. 28 represents a fifth-order polynomial. Each of its terms must be taken with the denominator and considered separately to determine compensation terms $f_{i}$ that are realizable, that is, they must not result in pure differentiation of any sensor signal. Defining the denominator of eqn. 28 as $\Delta$ for convenience and rewriting the numerator as a fifth-order polynomial gives:

$$
\begin{aligned}
\frac{A_{x}}{\delta_{b}}(s) & =\frac{K_{\delta_{b}}\left(a_{1} s+a_{0}\right)}{\Delta}+\frac{K_{\delta_{b}}\left(a_{2} s^{2}\right)}{\Delta} \\
& +\frac{K_{\delta_{b}}\left(a_{3} s^{3}\right)}{\Delta}+\frac{K_{\delta_{b}}\left(s^{5}+a_{4} s^{4}\right)}{\Delta}
\end{aligned}
$$

Now each of these terms can be equated respectively with the terms of eqn. 22 to determine the filters $f_{i}$. For example, for the pitch rate term:

$$
\begin{aligned}
f_{q}(s) & =\frac{K_{\delta_{b}}\left(a_{3} s^{3}\right)}{s(s+b)\left(s-X_{u}\right)[\zeta ; \omega]}\left(\frac{q}{\delta_{b}}(s)\right)^{-1} \\
& \approx \frac{K_{\delta_{b}} a_{3} s}{M_{\delta_{b}}(s+a)}
\end{aligned}
$$

$X_{u}$ was included for completeness until eqn. 31 , where it has been approximated as zero. This is reasonable since for the Apache it was flight identified to be $-0.02 \mathrm{sec}^{-1}$. Thus, the pitch rate filter is a first-order washout. Repeating the process for each sensor input, the total cue drive law is then:

$$
\begin{aligned}
A_{x}(s) & =\frac{K_{\delta_{b}}\left(a_{1} s+a_{0}\right)}{-M_{\delta_{b}} g(s+a)} \dot{x}(s)+\frac{K_{\delta_{b}} a_{2} s}{M_{\delta_{b}}(s+a)} \theta(s) \\
& +\frac{K_{\delta_{b}} a_{3} s}{M_{\delta_{b}}(s+a)} q(s)+\frac{K_{\delta_{b}} s^{2}\left(s+a_{4}\right)}{(s+b)[\zeta ; \omega]} \delta_{b}(s)
\end{aligned}
$$

Based on iterative examination of the cue controllability and the resulting trajectory response and on preliminary piloted evaluations, the zeros $z_{1}$ and $z_{2}$ were chosen to be equal at $-1.765 \mathrm{rad} / \mathrm{sec}$. Once these were selected, the numerator polynomial could be computed. Finally, the gain $K_{\delta_{b}}$ was chosen such that $f_{\dot{x}}(s)$ has a steady state value of $K_{\dot{x}}$, so that in the steady state the cue would rest at the tip of the velocity vector. Thus, the cue response transfer function was:

$$
\frac{A_{x}}{\delta_{b}}(s)=\frac{-2.21(s+1.765)(s+1.765)(s+0.262)}{s(s+.399)(s+0.02)}
$$

The following represents the corresponding display law that was evaluated in the simulation:

$$
\begin{aligned}
A_{x}(s) & =K_{\dot{x}}\left[\frac{1.42 s+0.262}{s+0.262} \dot{x}(s)-59.3 \frac{s}{s+0.262} \theta(s)\right. \\
& -32.1 \frac{s}{s+0.262} q(s) \\
& \left.-2.15 \frac{s^{2}(s+9.36)}{(s+0.399)[0.805 ; 3.46]} \delta_{b}(s)\right]
\end{aligned}
$$

where now the display gain $K_{\dot{x}}$ has been factored out so that the terms in brackets are in physical units of $\mathrm{ft} / \mathrm{sec}$.

\section{Lateral Axis Design}

A similar design procedure is followed for the lateral axis, but it is less complex because of the simpler vehicle response in this axis:

$$
\frac{\phi}{\delta_{a}}(s)=\frac{L_{\delta_{a}}}{s[\zeta ; \omega]}
$$

This leads to a fourth-order numerator for the cue response transfer function:

$$
\frac{A_{y}}{\delta_{a}}(s)=\frac{K_{\delta_{a}}\left(s+z_{1}\right)\left(s+z_{2}\right)[\zeta ; \omega]}{s\left(s-Y_{v}\right)[\zeta ; \omega]}
$$

which is then distributed among the sensor signals. Unlike $X_{u}$, the derivative $Y_{v}$ cannot be cancelled with a numerator 
free $s$, since it was flight identified to be $-0.279 \mathrm{sec}^{-1}$. The resulting form for the lateral cue law is then:

$$
\begin{aligned}
A_{y}(s) & =\frac{K_{\delta_{a}} a_{0}}{L_{\delta_{a}} g} \dot{y}(s)+\frac{K_{\delta_{a}} a_{1} s}{L_{\delta_{a}}\left(s-Y_{v}\right)} \phi(s) \\
& +\frac{K_{\delta_{a}} a_{2} s}{L_{\delta_{a}}\left(s-Y_{v}\right)} p(s) \\
& +\frac{K_{\delta_{a}} s^{2}\left(s+a_{3}\right)}{\left(s-Y_{v}\right)[\zeta ; w]} \delta_{a}(s)
\end{aligned}
$$

Again, after iterative examination to optimize the trajectory response, the two zeros and the gain $K_{\delta_{a}}$ were set such that the cue response transfer function for piloted evaluation was:

$$
\frac{A_{y}}{\delta_{a}}(s)=\frac{2.77(s+2.026)(s+2.026)}{s(s+0.279)}
$$

and the drive equation was:

$$
\begin{aligned}
A_{y}(s) & =K_{\dot{y}}\left[\dot{y}(s)+40.5 \frac{s}{s+0.279} \phi(s)\right. \\
& +18.2 \frac{s}{s+0.279} p(s) \\
& \left.+2.69 \frac{s^{2}(s+9.05)}{(s+0.279)[0.582 ; 4.29]} \delta_{a}(s)\right]
\end{aligned}
$$

\section{Display Laws Based on Performance Design}

The third design, based on a methodology developed in Ref. 4, is referred to as the "performance" design. It seeks to ensure good task performance but is balanced by pilot workload considerations. Besides this difference in emphasis, the workload and performance designs differ in the sensor signal distribution used to achieve the desired frequency response characteristics of the cue.

This method begins by selecting a desired transfer function of the vehicle's velocity response to be achieved when the pilot closes the control loop via the display. These dynamics represent how the velocity vector on the display would respond to the pilot maintaining the cue position at a fixed distance from the reticle (i.e., when the pilot is trying to establish a desired horizontal velocity). From Figure 2, if the pilot raises his gain high enough in the inner loop, then

$$
\frac{\delta_{b}}{P_{x}}(s) \approx \frac{\delta_{b}}{A_{x}}(s)
$$

Consequently, the inverse of the cue-to-stick dynamics may be used as series equalization with the open-loop, potentially poor vehicle velocity and position dynamics. If a desired vehicle velocity transfer function is selected, the cueto-stick transfer function is

$$
\frac{A_{x}}{\delta_{b}}(s)=\left.K_{\dot{x}} \frac{\dot{x}}{\delta_{b}}(s)\right|_{\text {Aircraft }} \times\left.\left(\frac{\dot{x}}{\dot{x}_{c}}(s)\right)^{-1}\right|_{\text {Desired }}
$$

since $A_{x}$ is the pilot commanded velocity. The denominator of the cue-to-stick transfer function contains the dynamics of the open-loop aircraft so that when it is inverted by the pilot's high gain, the open-loop dynamics are effectively cancelled. These cancelled dynamics are replaced by the desired closedloop velocity dynamics that are achieved when the pilot is controlling the vehicle in response to cue position errors.

For the AH-64, the velocity dynamics are (neglecting the identified delay from eqn. 1 and using eqn. 25)

$$
\frac{\dot{x}}{\delta_{b}}(s)=\frac{-2.49 g(s+0.262)}{s(s+0.02)(s+0.399)[0.805 ; 3.46]}
$$

In order for $A_{x} / \delta_{b}$ to have a gain-like response at high frequencies, its numerator and denominator should be of the same order. Thus, the desired $\dot{x}_{c} / \dot{x}$ transfer function should be 4 th over a 0 th order. To prevent any velocity overshoot in the desired response, all of the roots in the desired velocity transfer function were placed on the real axis in the complex plane. The four equal roots were selected at -2.5 $\mathrm{rad} / \mathrm{sec}$. The selection of these roots is empirical but is based on some important points. First, the roots should be selected such that the high frequency gain of the cue to pilot inputs (of eqn. 41 ) is within a desired sensitivity range. If the roots of the desired velocity transfer function are all at low frequency, the high-frequency gain will be too high for a given velocity vector scaling gain. Second, the roots should be at a low enough frequency so that some immediate response to stick input occurs in the $1-10 \mathrm{rad} / \mathrm{sec}$ range. Third, as the roots move lower in frequency, the gains on the feedback signals in the display laws tend to increase.

For the design in this experiment,

$$
\frac{A_{x}}{\delta_{b}}(s)=\left.K_{\dot{x}} \frac{\dot{x}}{\delta_{b}}(s)\right|_{\text {Aircraft }} \times \frac{(s+2.5)^{4}}{(2.5)^{4}}
$$

This controlled-element transfer function then needs to be distributed among the aircraft states rather than depending solely on pilot input. If the cue position is treated as the commanded velocity, $K_{\dot{x}} \dot{x}_{c}$, then

$$
\begin{aligned}
A_{x}(s)= & \left.K_{\dot{x}} \frac{\dot{x}_{c}}{\dot{x}}(s)\right|_{\text {desired }} \dot{x}(s) \\
= & K_{\dot{x}} \dot{x}+K_{\dot{x}}\left[\frac{(s+2.5)^{4}}{(2.5)^{4}}-1\right] \dot{x} \\
= & K_{\dot{x}} \dot{x}+1.6 K_{\dot{x}} \ddot{x}_{c o m p} \\
& +K_{\dot{x}}\left[\frac{s\left(s^{2}+10 s+37.5\right)}{(2.5)^{4}}\right] \ddot{x}
\end{aligned}
$$




$$
\begin{aligned}
= & K_{\dot{x}}\left[\dot{x}+1.6 \ddot{x}_{\text {comp }}+\frac{-2.49 g s(s+0.262)}{(2.5)^{4}(s+0.02)}\right. \\
& \left.\times \frac{\left(s^{2}+10 s+37.5\right)}{(s+0.399)[.805 ; 3.46]} \delta_{b}\right]
\end{aligned}
$$

In the steady state, the cue indicates the scaled velocity $K_{\dot{x}} \dot{x}$. A gained acceleration term and a 4 th over a 4 th order washout filter is on the stick. This high order filter indicates that a large portion of the cue response is generated from stick input, which is pure prediction based upon the known open-loop helicopter velocity response and a distributed portion of the desired velocity response. The simulation showed that the sensitivity of this stick term in the cue response for aircraft changes (across the vehicle operational weight and inertia envelope) was acceptable.

The development in the lateral axis is identical. Here the desired velocity roots are $-2,-2$, and $[0.582 ; 4.29]$. The complex zeros were chosen to cancel the high frequency lightly damped roll axis natural response in the $A_{y} / \delta_{a}$ transfer function. Otherwise, a slight oscillation at the underdamped roll mode would appear in the cue response to pilot input. This jitter was a problem early in the simulation, and the proper placement of the zeros eliminated it. Using the same development as in the longitudinal axis, the lateral axis cue response is

$$
\begin{aligned}
A_{y}(s)= & K_{\dot{y}}\left[\dot{y}+1.25 \ddot{y}_{c o m p}+\frac{6.32 g s}{(2)^{2}(4.29)^{2}(s+0.279)}\right. \\
& \left.\times \frac{\left(s^{2}+9.07 s+42.9\right)}{[0.582 ; 4.29]} \delta_{a}\right]
\end{aligned}
$$

The quantities $\ddot{x}_{\text {comp }}$ and $\ddot{y}_{\text {comp }}$ are complementary filtered values. They are comprised of low frequency accelerometer measurements and high-frequency attitude-rate imputs. This filtering attenuates vibratory accelerometer measurements and cuts off the immediate accelerations due to rotor flapping from stick inputs. These immediate accelerations contribute to noise and are not useful in the pilotvehicle-display crossover frequency range. The filters are

$$
\begin{aligned}
& \ddot{x}_{\text {comp }}=\frac{1}{s+1} \ddot{x}-\frac{g s}{(s+1)(s+0.02)} \dot{\theta} \\
& \ddot{y}_{\text {comp }}=\frac{1}{s+1} \ddot{y}+\frac{g s}{(s+1)(s+0.279)} \dot{\phi}
\end{aligned}
$$

Comparison of Display Laws and Task Performance Prediction

The analytical frequency responses for the four longitudinal-axis acceleration cues are presented in Figure 5. First it is seen that the performance and workload designs are nearly identical, though they were developed independently. The gain-like characteristics are apparent above about $2 \mathrm{rad} / \mathrm{sec}$. The other two designs roll off rapidly above this frequency. In the mid-frequency range around $1 \mathrm{rad} / \mathrm{sec}$, the performance and workload designs have roughly $\mathrm{K} / \mathrm{s}$ characteristics. The modified production design has more phase lag than the production design in the mid-frequency region, but has better damping characteristics as discussed in the design section. The lateral axis frequency responses, when plotted, show similar trends.

The effect of these differences on task performance can be shown analytically by again referring to Figure 2 . The pilot gain was set to $0.3 \mathrm{in} / \mathrm{deg}$, and the control limit was set to \pm 5 in. The selected pilot gain resulted in crossover frequencies in the inner loop of Figure 2 to be between 2 and $3 \mathrm{rad} / \mathrm{sec}$ for each display cue law. The position loop was closed for each design and then driven with a step position command of 10 feet. The resulting vehicle trajectory and the control inputs required to achieve those trajectories are shown in Figure 6 for all four cue designs. It is seen that the position trajectories for the workload and performance designs are well-damped and relatively smooth. The modified production design is damped but not as smooth, while the production design is oscillatory with undershoot. Regarding the control inputs, the workload and performance traces
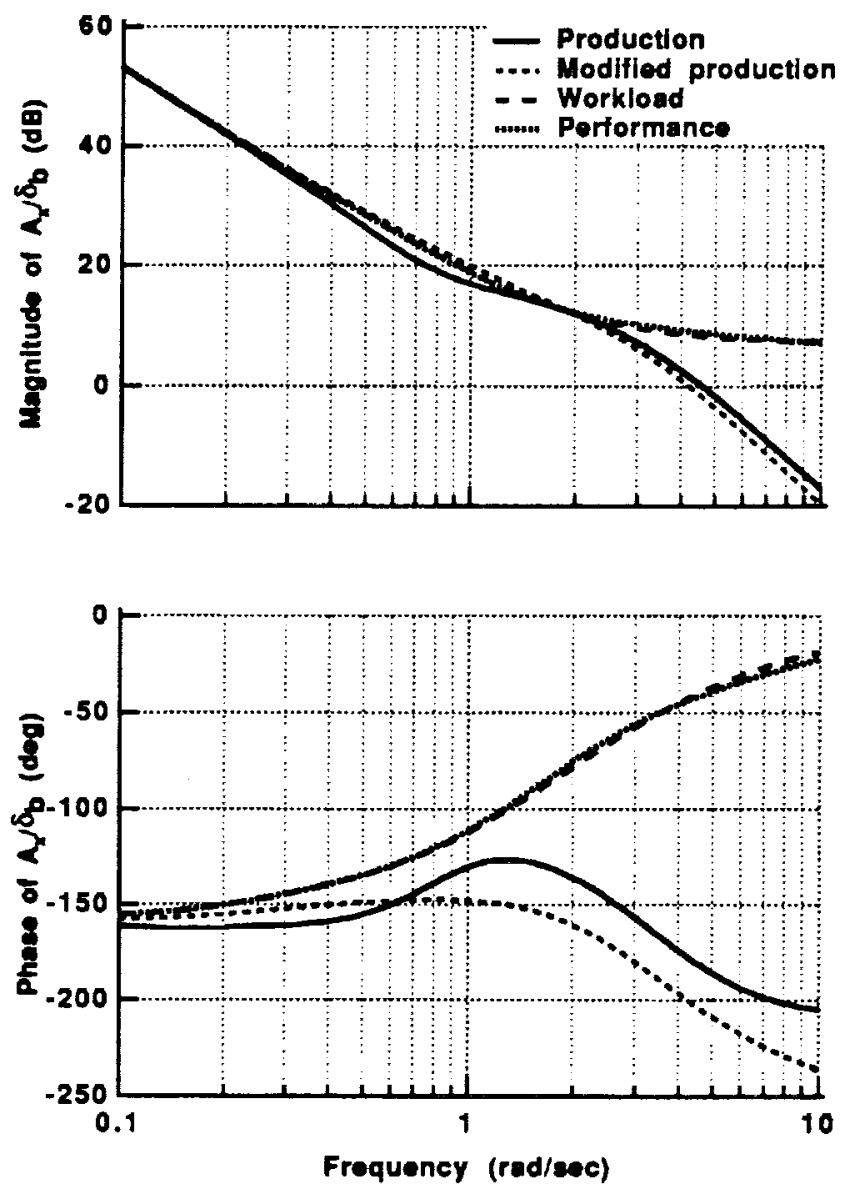

Fig. 5 Analytical frequency responses of four longitudinal cues. 
show one control reversal, the modified production design shows significant oscillation, and the production design has some oscillation and is generally complex.

Based on these analyses, it could be predicted that the workload and performance designs would yield both the best performance and lowest workload, the modified production design the third best performance and the production design the poorest performance. The piloted assessments of relative workload for the modified production and production designs is difficult to predict from the traces.

The Bode plots for the workload and performance designs show the gain-like characteristics extending indefinitely to high frequency. Although noise is generally not a factor in simulation, in a flight environment the cue response must be attenuated to prevent sensor and pilot controlinduced noise from passing through to the cue, causing it to jitter on the display. Therefore, for completeness of the experiment, a first-order $10 \mathrm{rad} / \mathrm{sec}$ filter was placed on the total cue displacements $A_{x}$ and $A_{y}$ before they were sent to the display. This was done for the performance and workload designs only, since the other designs already have highfrequency attenuation. While the filter adds phase lag to the
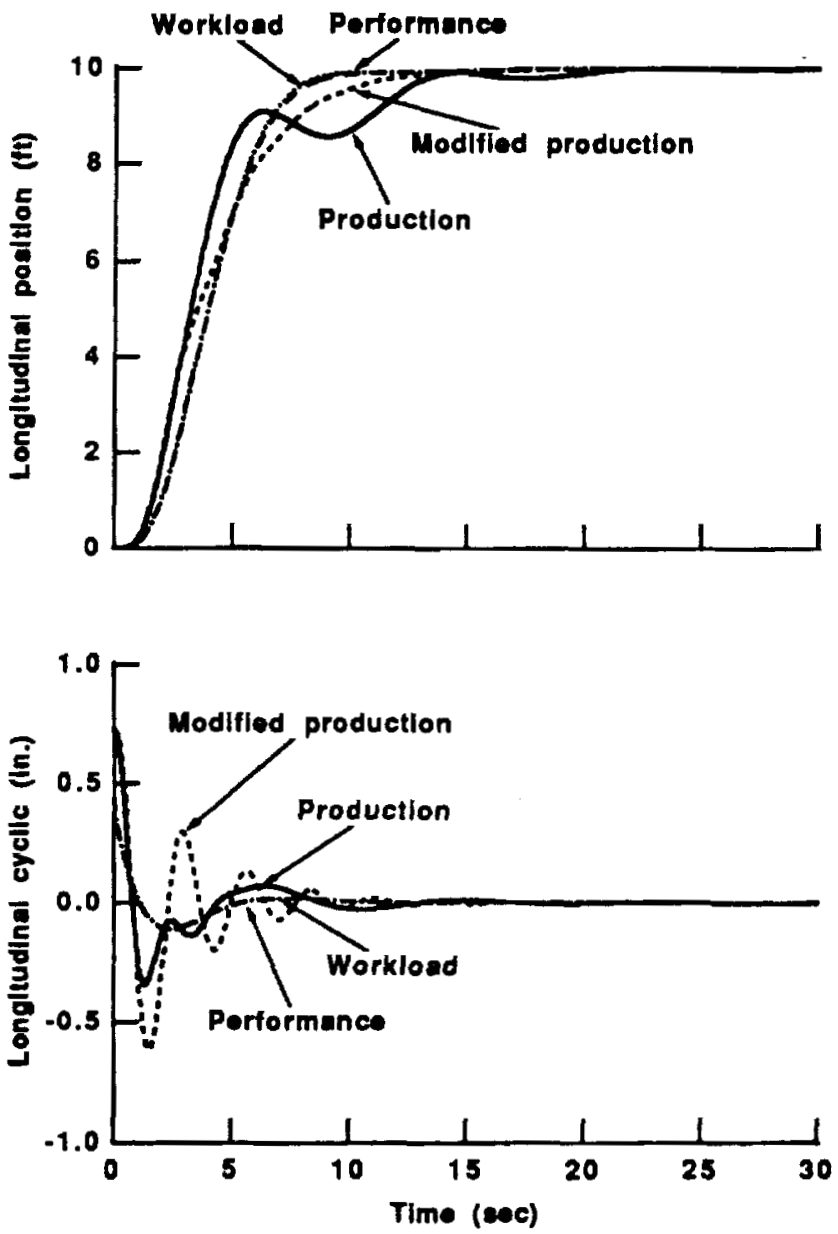

Fig. 6 Analytical position responses and control traces for four longitudinal cues. cue response, for these hover maneuvering tasks it does not appear to significantly degrade stability margins. The flight data analyzed in Ref. 2 exhibited measured crossover frequencies of $1-4 \mathrm{rad} / \mathrm{sec}$ with the same noise attenuation filter. Other display laws with similar high-frequency gains and the same noise attenuation filters have also been flown successfully (Refs. 1, 4, and 5).

\section{EXPERIMENT CONDUCT}

\section{Simulator Configuration}

The experiment was conducted on the NASA Ames Vertical Motion Simulator (VMS). The main objective was to perform piloted evaluations of the existing production display laws and the three new display designs to assess their impact on handling qualities, using both Apache-rated and non-Apache-rated test pilots. It was recognized that the validity of the results would be highly dependent on the simulation fidelity. Therefore, much attention was devoted to represent accurately the Apache using the simulator elements shown in Figure 7. This effort is described in detail in Ref. 10. To summarize, a nine-state ( 8 rigid body plus dynamic inflow) linear math model valid for the unaugmented AH-64 near hover was identified from flight data. A verified software representation of the AH-64 DASE was then added to the linear model. The aircraft rotorspeed and torque responses to collective were identified from flight data, to drive the cockpit and helmet-mounted displays. Significant effort was expended to identify also the static and dynamic characteristics of the AH-64's centerstick controller and pedals. These controller characteristics were used to tune the simulator's programmable control loaders. For added fidelity, a sound generator was matched qualitatively to an audio recording made within an Apache cockpit.

Because of the small displacements involved in the hover maneuvers, nearly the full potential of the VMS motion system could be used. At mid-to-high frequencies, 1:1 motion of the simulator with respect to the aircraft was achieved in all axes. In addition, the AH-64 Integrated Helmet and Display Sighting System (IHADSS) flight hardware was used (Figure 8). A simulated forward looking infrared (FLIR) image was shown on the helmet monacle, and the Apache Bob-Up mode symbology was superimposed on it. The FLIR and symbology images were made to match the written specifications and a video record from an AH- 64 in terms of symbology placement, size, scaling, and display feld-of-view. The total throughput time delay from control input to motion and visual response was matched as closely as possible to the flight-identified values for each axis. Pilot acceptance of the simulator as representative of an AH-64 was generally positive, as described in Ref. 10.

Piloted Tasks

Two tasks were developed to compare the display laws. In each, the pilot was advised to perform the task using the strategy of minimizing the acceleration cue error from the 


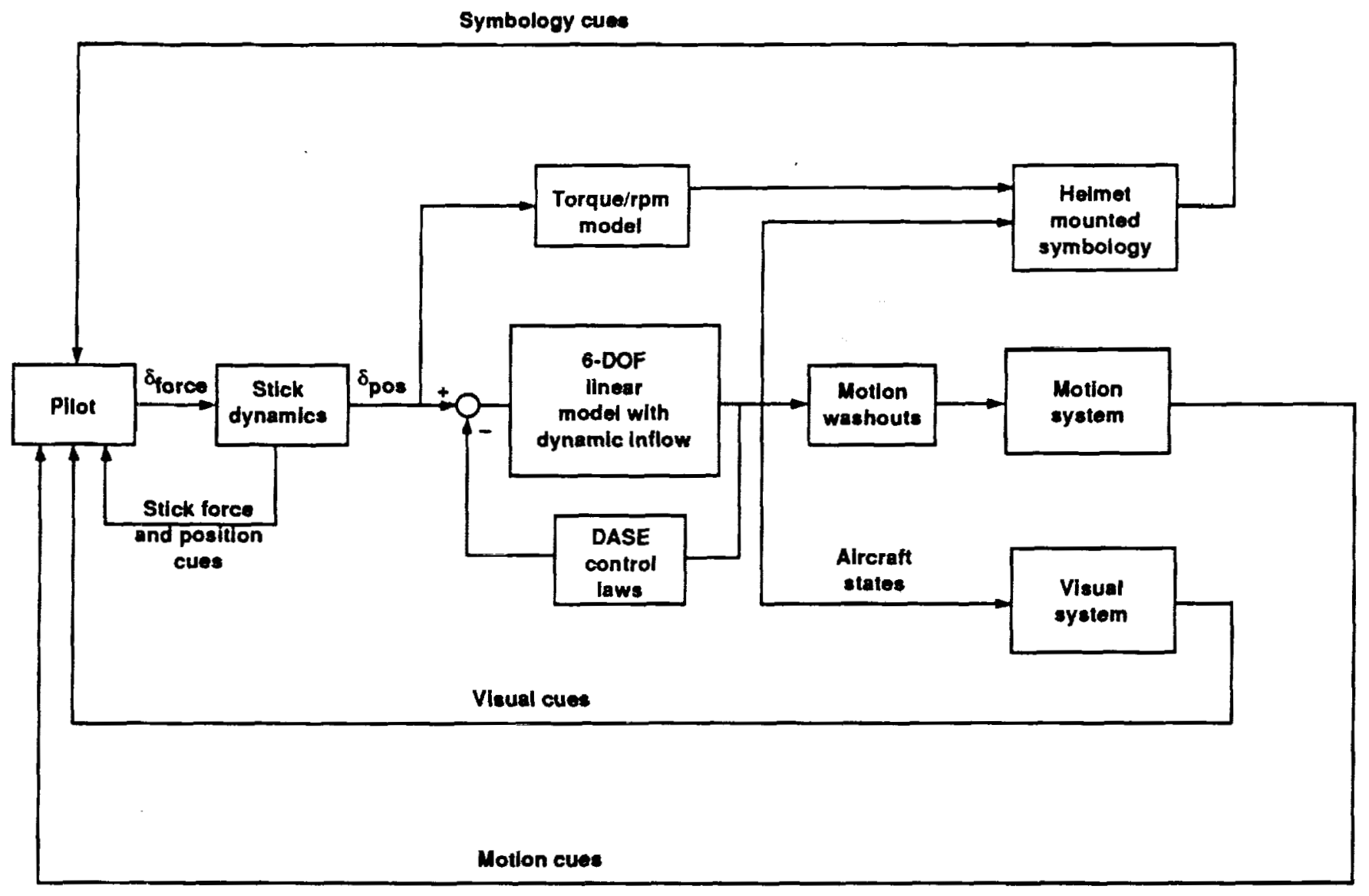

Fig. 7 AH-64 simulation components.

hover box. This strategy is the one taught to operational pilots. The first task, known as the pad capture, was to acquire the hover box from a diagonal $56 \mathrm{ft}$ offset ( $40 \mathrm{ft}$ each laterally and longitudinally) within 15 secs. In each run, the task was repeated four times; every 15 secs, the hover box was repositioned in earth axes $56 \mathrm{ft}$ diagonally forward or rearward of its last position. The objective of the task was to achieve a stable hover over the box before it was moved to the new position. The standards for desired performance were: 1) achieve position over/undershoot of less than one hover box width; 2) maintain altitude at $40 \pm 10 \mathrm{ft} ; 3$ ) maintain initial heading \pm 10 deg. The standards for adequate performance were twice those for desired. This task was meant to expose issues associated with the cue controllability and the position trajectories.

The second task was a Bob-Up/Bob-Down maneuver, in which the pilot began in a hover at $40 \mathrm{ft}$, ascended to a $70 \mathrm{ft}$ target altitude, then immediately descended to $40 \mathrm{ft}$ again. The objective was to perform the task in 15 secs while maintaining position over the hover box. The standards for desired performance were: 1) achieve target altitudes with over/undershoot less than $10 \mathrm{ft} ; 2$ ) maintain heading \pm 5 deg; 3) maintain position within the hover box. The standards for adequate performance were twice those for desired. This task was designed to compare the regulation capabilities of each cue during off-axis inputs.

The tasks were conducted using a baseline level of Dryden turbulence that was termed very light. Its root-meansquare (rms) magnitude was $0.3 \mathrm{f} / \mathrm{sec}$. Three pilots evaluated the display laws in the pad capture task under a light-tomoderate turbulence level (rms of $1.5 \mathrm{ft} / \mathrm{sec}$ ) to investigate potential disturbance rejection differences among the laws.

\section{Outside Visual Scene}

The pilot's visual information was presented using the AH-64 IHADSS monacle, which displayed the symbology superimposed on a simulated FLIR image of the outside world. The outside view was a head-tracked computergenerated scene. The offset of the FLIR turset from the pilot station was represented. The scene objects were adjusted in color to present a nighttime FLIR-like image once they were sent to the monacle display. Both white-hot and black-hot FLIR modes were available to the pilot. The monacle field of view was 40 Horiz. $\times 30$ Vert. degrees, while the simulated sensor field-of-regard was 240 Horiz. x 90 Vert. degrees.

The pad capture task was flown over a flat area with grid lines at ten foot intervals. The grid lines provided strong heading cues and some position cueing. The bob-up task was 


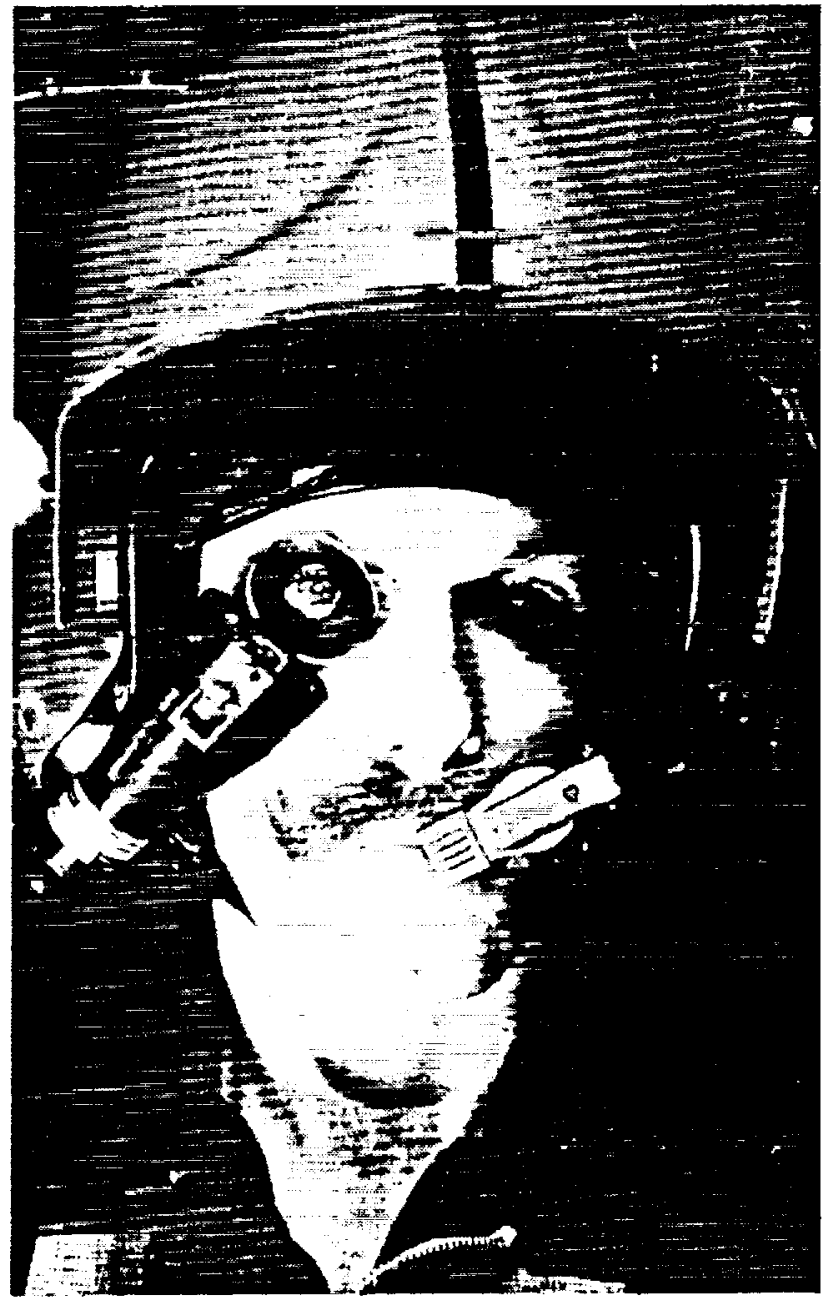

Fig. 8 AH-64 Integrated Helmet and Display Sighting System.

flown over a hover pad area with trees in the near field that provided some altitude cues.

While the simulated FLIR imagery was judged by the pilots to be reasonable in terms of object light intensity, all the pilots felt that the lack of texture and other fine detail made the outside cues far less useful than those of an actual FLIR. This, in combination with the symbology-oriented nature of the tasks and the nominal altitude used (40 ft), forced the pilots to rely more on the symbology than normally would be the case in reality. Some pilots estimated that they used the symbology for $90 \%$ of the cuing. Consequently, they were prevented from compensating for poor symbology drive laws by using outside cues, thus perhaps more clearly exposing the differences among the laws. Several Apache pilots stated that this poor-FLIR environment was similar to using the IHADSS at night during high hover operations, where significant ground cues are lost.

\section{Off-Nominal Configurations}

The new display laws were designed for a nominal aircraft configuration, namely the one used for the parameter identification flight tests that yielded the simple DASE-on transfer function models. The laws were then evaluated in the piloted simulation using the nine-state model with the DASE programmed explicitly. The simulation model had been identified for the same nominal aircraft weight and stores configuration as the display design model. To assure that the new display laws were not overly tuned to one aircraft configuration, the nine-state simulation model's parameters were varied to represent a light and a heavy stores configuration about the nominal. The pad capture task was performed by several pilots at these off-nominal conditions.

Test Pilot Participants

A total of ten experienced test pilots participated in the simulation as evaluators. Among them were four Apachequalified pilots, including: one instructor pilot from AQTD with over 700 hrs in the Apache and over 400 hours using the PNVS; one from the AQTD with 150 PNVS hours; one from the Aeroflightdynamics Directorate (AFDD) with 25 PNVS hours; and one from the manufacturer, McDonnell Douglas Helicopter Co., with 200 PNVS hours. The non-Apache rated pilots included two from NASA Ames, one from AFDD (with 30 PNVS hours), one from Sikorsky Aircraft (with helmet-mounted display experience), one from Boeing Helicopters, and one from the Navy Test Pilot School.

\section{Piloted Evaluations}

Each pilot was allowed to practice the tasks with all four of the cues until he felt that his performance had stabilized. Several training sessions were generally required. He then completed formal evaluations of all the cues for one task with one aircraft and turbulence configuration. He was not informed of which cue he was evaluating. The order that the cues were presented was varied for each evaluation session. For any one task, the procedure was to finish a session with a re-evaluation of the cue flown first, to see if learning effects were a factor.

\section{Data Collection}

Data collected during evaluations comprised statistical and time history data to document task performance, verbal answers to a questionnaire, and Cooper-Harper pilot ratings (Ref. 11).

\section{RESULTS}

\section{Task Performance Results}

Figure 9 presents positioning performance crossplots for all pilots conducting four pad captures each for each acceleration cue. In terms of deviation from a $45^{\circ}$ horizontal path, the trajectories are seen to be more accurate and more consistent for the workload and performance designs in comparison with both the production and modified production designs.

Figure 10 presents the acceleration cue error from the hover box for the same runs. Since the pilot was advised to place and keep the cue on the box during the acquisition, 

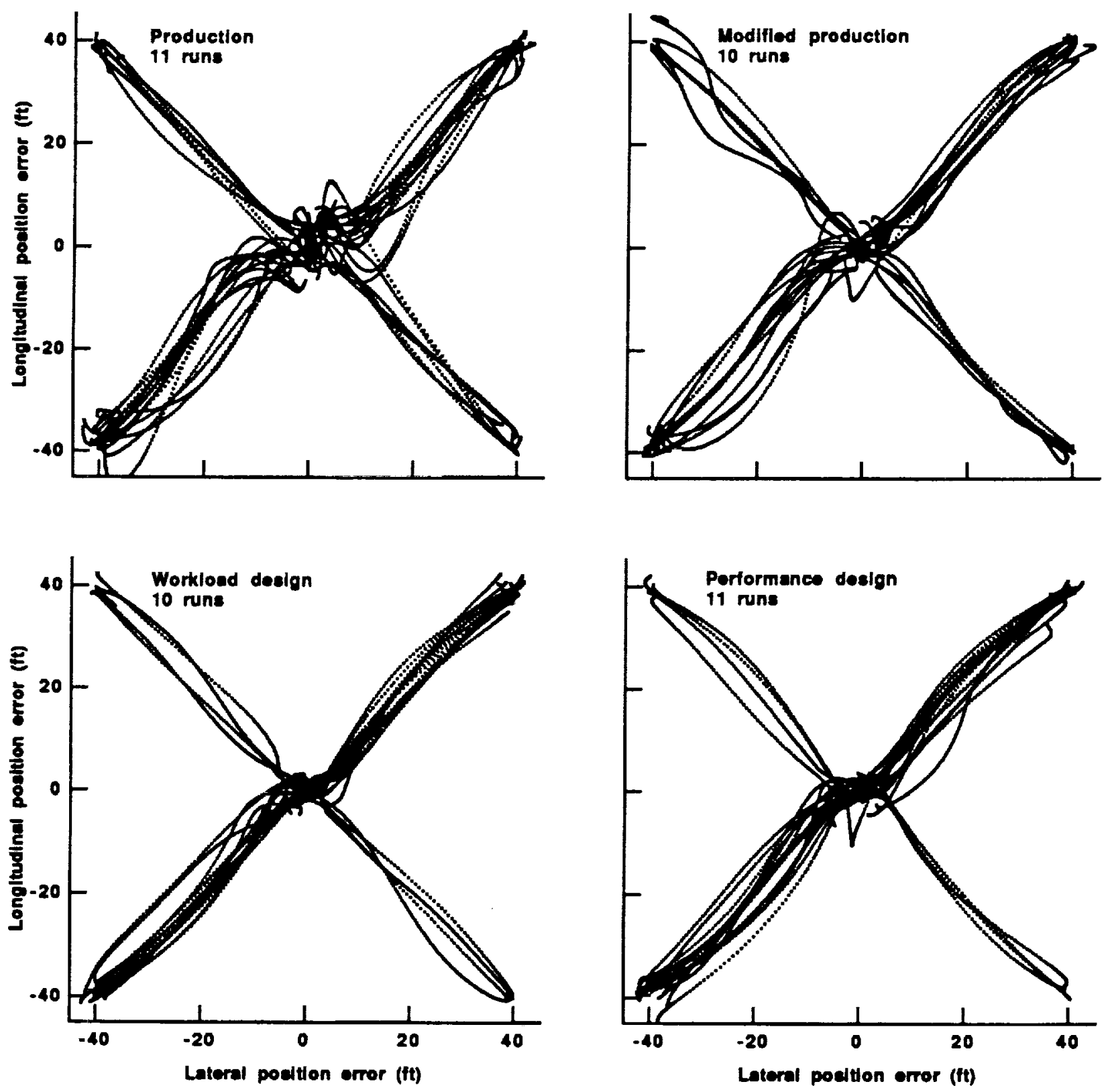

Fig. 9 Position crossplots for four cues, pad capture task.

these plots indicate cue controllability and are thus a measure of workload. The workload and performance designs show a narrower concentration of points along a $45^{\circ}$ path and at the origin, indicating lower workload in comparison with the other two.

The altitude performance for four evaluation runs by one pilot is presented in Figure 11. While all the traces remain in the desired performance region, the production and modified production traces exhibit large oscillations that appear nearly divergent compared with the more damped traces for the workload and performance designs. The differences suggest that the improved controllability of the workload and performance cues allowed the pilot to devote more attention to scanning the altitude tape, thus better controlling the altitude.

As a check of the analytical performance predictions described earlier, Figure 12 presents longitudinal trajectory and control input time histories from analysis and from simulation for a $20 \mathrm{ft}$ longitudinal capture using the performance design. The position trajectories are in good agreement except for pilot and system time delays that were not modeled in the analysis. The simulation control input trace shows a higher frequency component superimposed on a trend that generally matches the analysis. This "dither" may result from the pilot's uncertainty about how much control is required to move the cue to the box. 

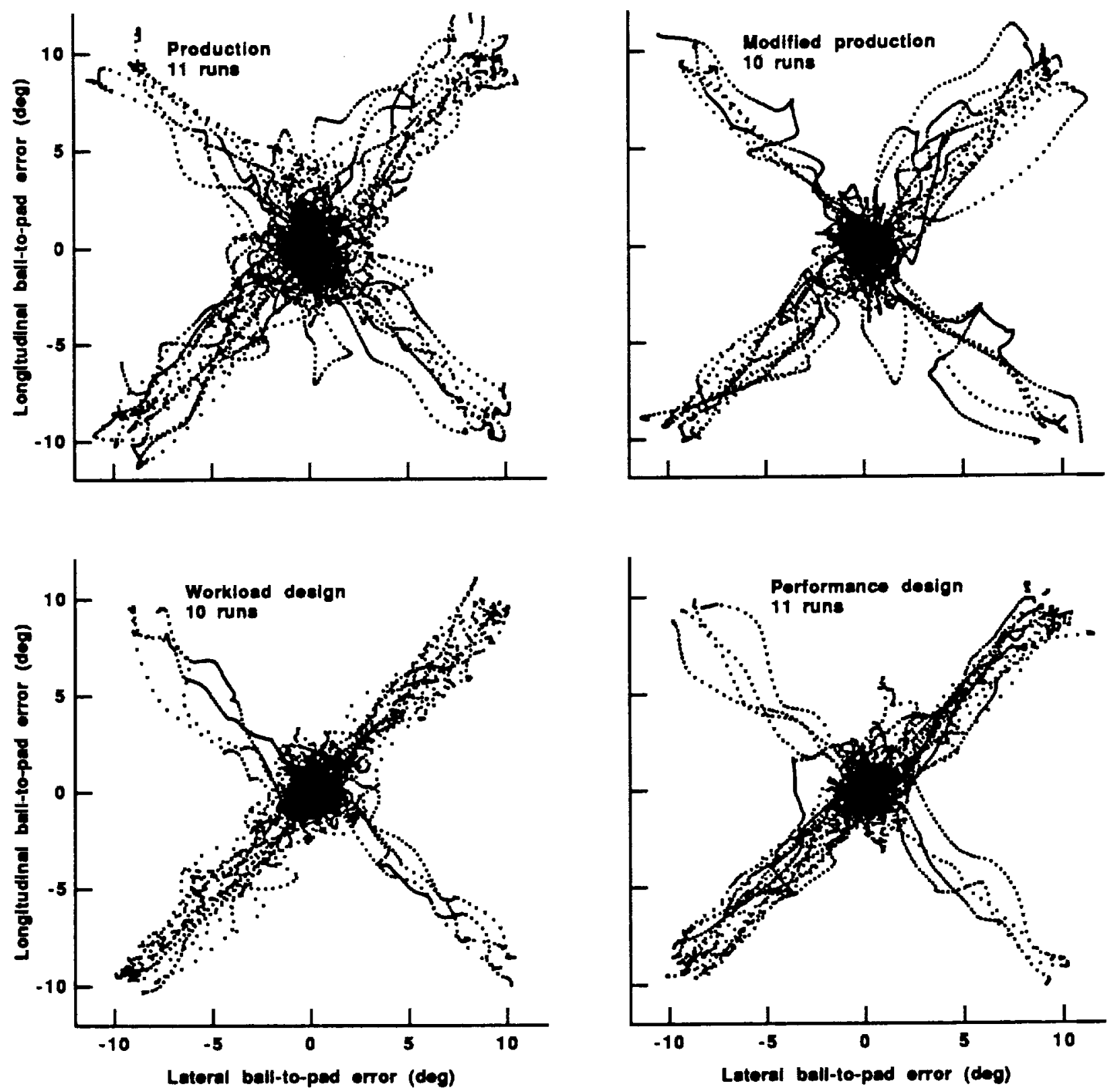

Fig. 10 Cue-to-box error crossplots for four cues, pad capture task.

The performance measure of interest for the bob-up task is the horizontal position error during the vertical maneuver. Figure 13 presents the root-mean-square position errors seen for the bob-up task as a function of cue drive law. Each point represents an individual bob-up maneuver. The modified production law has the lowest position error, followed by the workload, performance, and production laws. The most likely reason for this trend is that since the performance and workload laws use pilot input as one sensor for the cue, the high-frequency part of the cue motion is due to the control rather than to any actual aircraft movement. Thus, less aircraft motion is required to keep the cue on the box than for the production and modified production laws. While the pilot workload is reduced, for these small inputs the positioning performance may be slightly degraded.

Pilot Rating Results

Figure 14 presents a compilation of all the pilot ratings for the pad capture task in the baseline turbulence, nominal weight configuration. All the rating means fall in the Level 2 region. According to pilot comments given during the rating procedure, the workload associated with flying the ratedamped aircraft using a narrow field-of-view display with simulated FLIR imagery made the vehicle-display system unsatisfactory without improvement. The workload associated with control of the vertical axis, which required frequent 


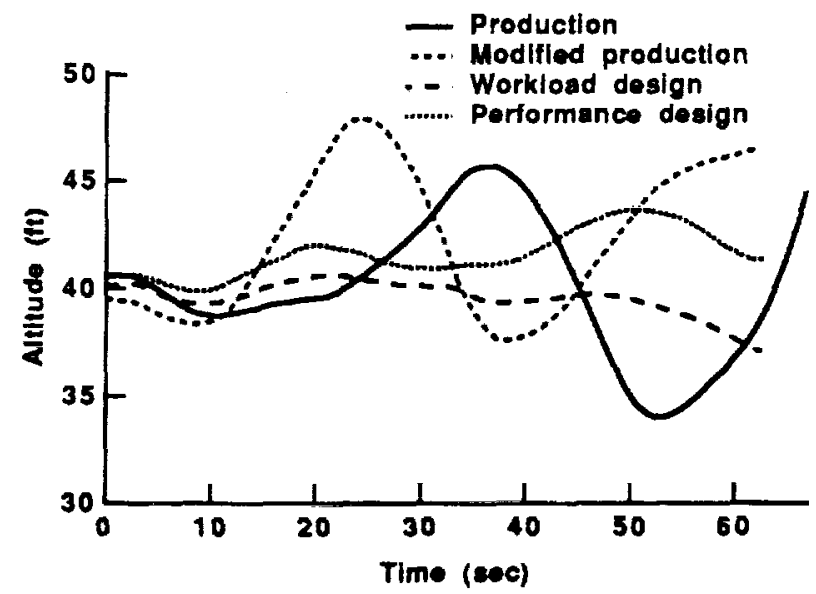

Fig. 11 Altitude performance for four cues during four pad captures.
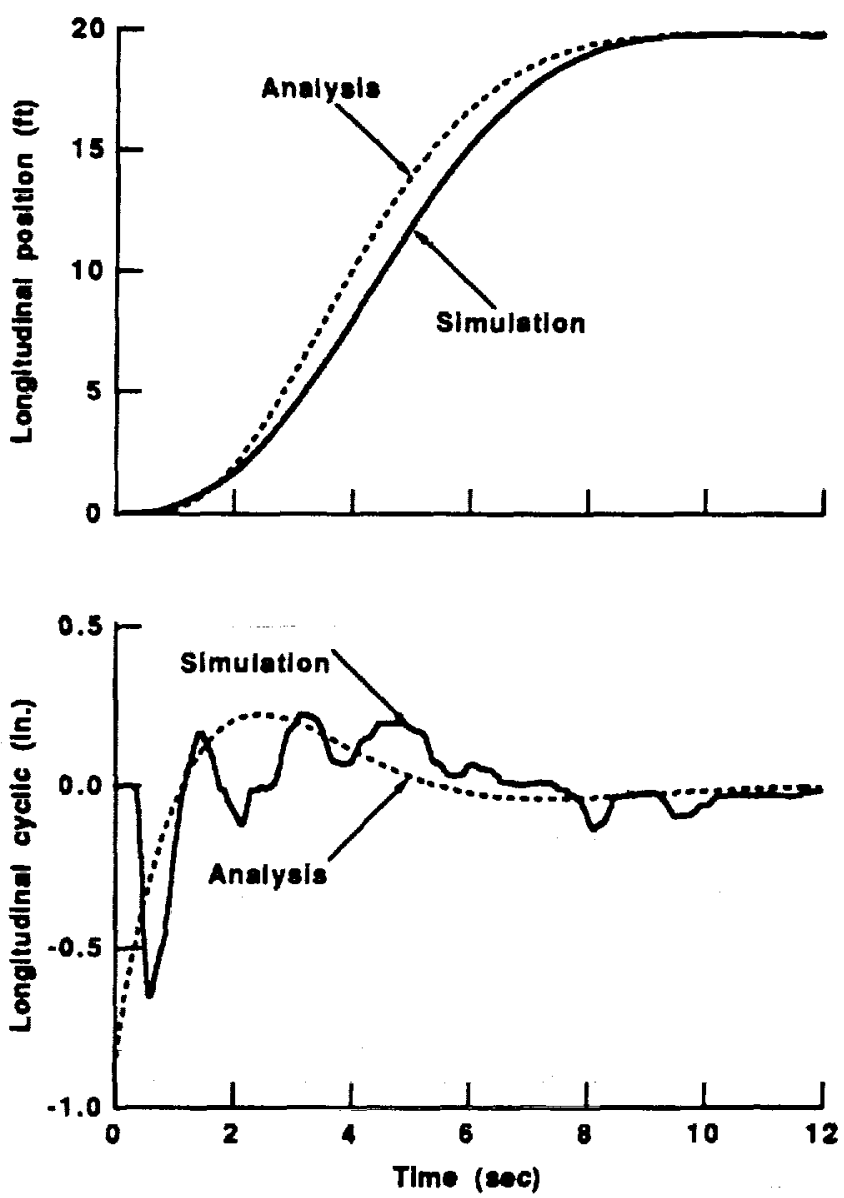

Fig. 12 Evaluation of pilot-vehicle-display model for performance design.

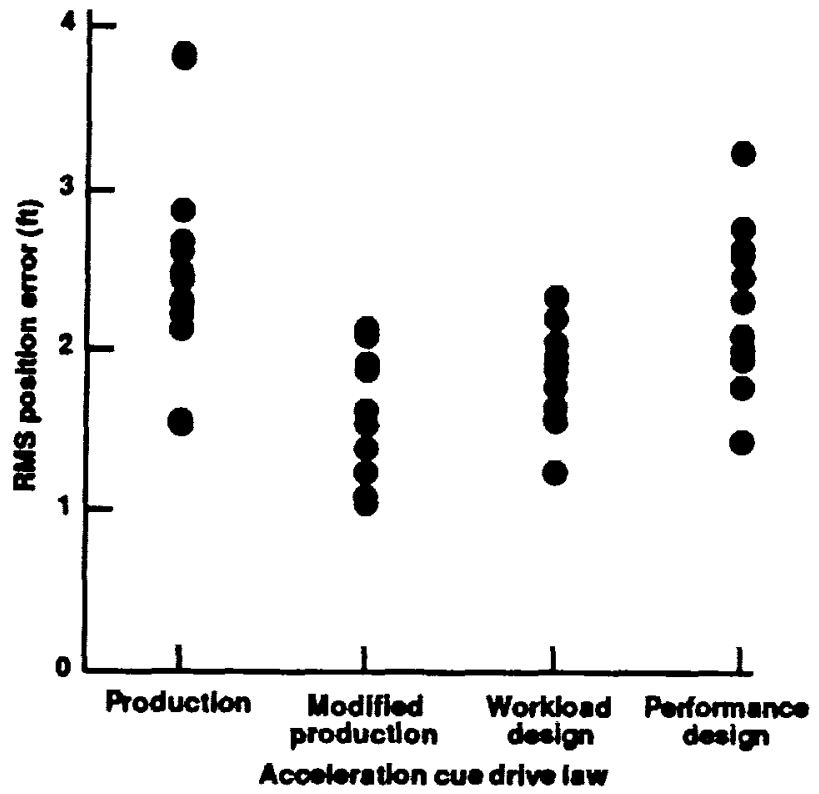

Fig. 13 Positioning performance for four cues, bob-up task, all runs.

scanning away from the central symbology to the altitude tape, also was frequently sighted as a factor contributing to the Level 2 ratings.

However, there are significant differences among the cue drive laws. The mean rating improves from 5.9 for the production law to 4.3 for the workload design, which had a slightly better mean than the performance design. This improvement reflects a reduction in pilot compensation requirements from "extensive" to "moderate" to perform the task. It is important to note that the $90 \%$ confidence bars (Ref. 12) do not overlap for the best versus the worst display configurations. Moreover, each of the ten pilots assigned a better rating to the workload and performance designs than to the production laws.

Figure 15 presents the rating data for the the bob-up task at nominal weight and baseline turbulence. Again, the workload design received the best ratings, followed by the performance and then the production and modified production designs.

\section{Summary of Pilot Comments}

Following is a summary of the pilot comments for all the cue laws tested. They are extracted from answers given verbally in response to a questionnaire after every evaluation rin.

Pilot comments conceming the production law indicated that the cue was unpredictable and difficult to control. A large amount of effort was required to keep the cue within the hover box. In the pad capture task, the cue was said to cause pilot-induced oscillations (PIO's) unless the task aggressiveness was reduced. Over- and undershoots in position were seen with the cue. The workload to control the cue 


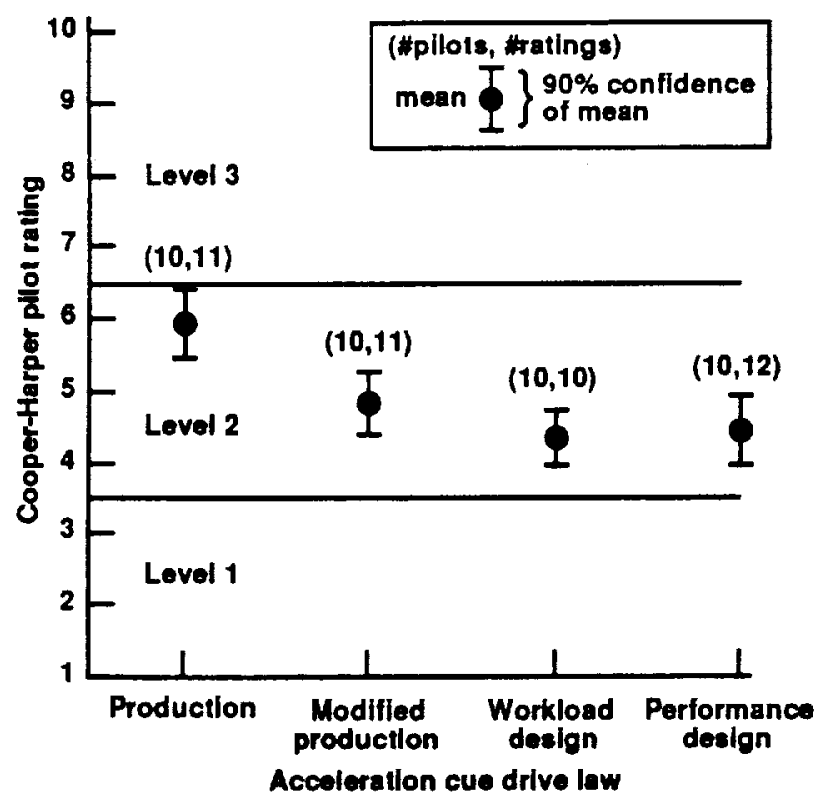

Fig. 14 Mean and $90 \%$ confidence values for all ratings, pad capture task.

allowed less time for crosschecking the altitude and heading, degrading performance in those axes. For the bob-up task, the attention required to maintain the cue on the box detracted from the altitude performance.

The modified production law was considered an improvement over the production law in controllability and positioning performance. It was still judged unpredictable,

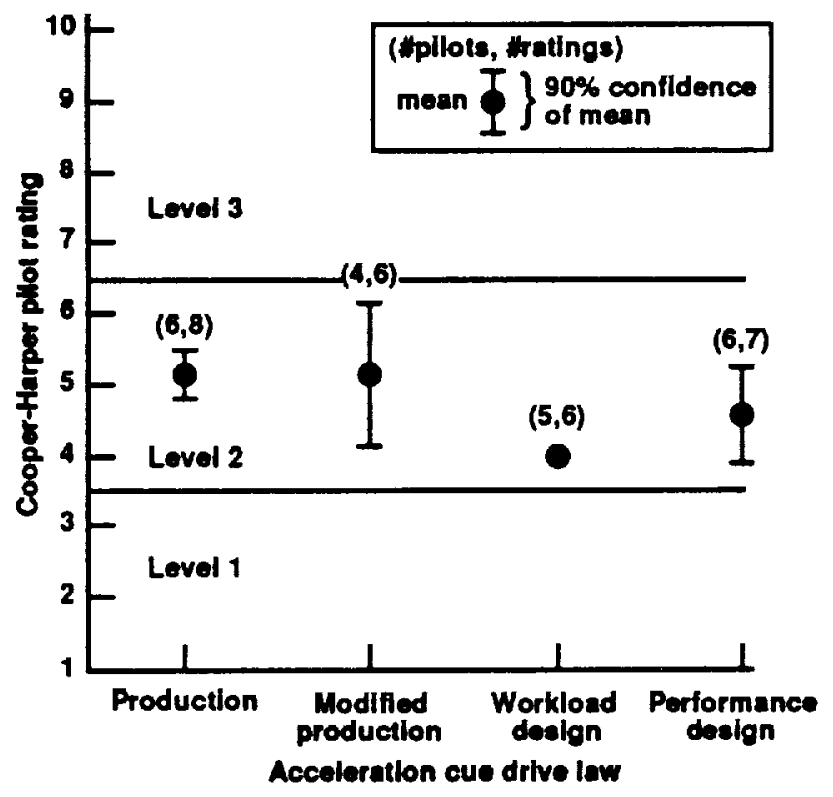

Fig. 15 Mean and $90 \%$ confidence values for all ratings, bob-up task. sluggish, and slightly prone to PIO. However, more attention was available to scan the altitude and heading for both tasks.

The workload design was described as very predictable and easily controllable. It allowed more aggressiveness and was felt by the pilots to allow much improved position and velocity performance. There were no PIO tendencies, and the workload was reduced significantly. Thus, there was substantially more attention available for scanning and control of the altitude and heading. These improvements were apparent for both tasks. Pilots noted that the cue sometimes appeared to have a slight overshoot in response to a quick control input, which they referred to as jitter. However, the effect was not judged objectionable. All the AH-64 rated pilots noted that they had no trouble adjusting to the characteristics of this new law.

Comments on the performance design were very similar to those for the workload design, except that no cue jitter was noted. The position trajectories for the pad capture task were described as nicely convergent. There was a wider dispersion of ratings and a slightly worse mean rating with this design for both tasks. The difference in ratings for the bob-up task seems to correlate with the task positioning performance presented in Figure 13. Recall that the performance design assigns more of the cue response to the control input than does the workload design, which may degrade its regulation performance.

\section{CONCLUSIONS}

A piloted simulation was conducted to investigate handling qualities improvements attainable through the application of improved display laws for hover maneuvering, using FLIR imagery with superimposed symbology. Three new display law methods were applied to the AH-64 Apache and compared with its existing display laws. The new laws, termed the modified production, performance, and workload designs, were compared analytically, and then tested using a pilot-in-the-loop simulation that was extensively validated and well accepted by the pilots. The analytical comparisons showed an improvement in both performance and workload for the new laws. These analytical improvements were confirmed in the piloted evaluations by ten test pilots, four of whom were AH-64 rated. The new performance and workload laws, which use stick position to achieve an immediate response of the acceleration cue to pilot input, were determined to benefit significantly handling qualities in comparison with the production and modified production laws. First, the new laws yielded improved performance for the horizontal positioning primary task, while allowing more attention for improved performance in secondary tasks such as altitude regulation. Second, the new laws elicited favorable pilot comments; all ten pilots said they preferred the new laws over the existing laws. Finally, all ten pilots assigned a better pilot rating to each of the new laws than to the existing laws. 


\section{REFERENCES}

${ }^{1}$ Eshow, M. M., Aiken, E. W., and Hindson, W. S.,"Preliminary Results of a Flight Investigation of Rotorcraft Control and Display Laws for Hover," American Helicopter Society National Specialists' Meeting in Flight Controls and Avionics, Cherry Hill, New Jersey, October 1987.

${ }^{2}$ Eshow, M. M., "Flight Investigation of Variations in Rotorcraft Control and Display Dynamics for Hover," Journal of Guidance, Control, and Dynamics, Vol. 15, No., 2, 1992, pp. $482-490$.

${ }^{3}$ Tsoubanos, C. M., and Kelley, M. B., "Pilot Night Vision System (PNVS) for Advanced Attack Helicopter (AAH)," Proceedings of the 34th Annual National Forum of the American Helicopter Society, Washington, D. C., 1978.

${ }^{4}$ Schroeder, J. A., and Merrick, V. K., "Flight Evaluations of Several Hover Control and Display Combinations for Precise Blind Vertical Landings," Journal of Guidance. Control, and Dynamics, Vol. 15, No. 3, 1992, pp. 751-760.

${ }^{5}$ Schroeder, J. A., Eshow, M. M., and Hindson, W. S., "An In-Flight Investigation of Display Drive Law Improvements to an Operational Attack Helicopter," Proceedings of the 46th Annual National Forum of the American Helicopter Society, Washington, D. C., 1990.

${ }^{6}$ Weir, D. H., Klein, R. H., and McRuer, D. T., "Principles for the Design of Advanced Flight Director Systems
Based on the Theory of Manual Control Displays," NASA CR-1748, 1971.

${ }^{7}$ Tischler, M. B., and Cauffman, M. G., "FrequencyResponse Method for Rotorcraft System Identification with Applications to the BO-105 Helicopter," Proceedings of the 46th Annual National Forum of the American Helicopter Society, Washington, D. C., 1990.

${ }^{8} \mathrm{Ham}$, J. A., Butler, C. P., "Flight Testing the Handling Qualities Requirements of ADS-33C - Lessons Learned at ATTC," Proceedings of the 47th Annual National Forum of the American Helicopter Society, Phoenix, AZ, 1991.

${ }^{9}$ McRuer, D. T., and Krendel, E. S., "Mathematical Models of Human Pilot Behavior," AGARDograph No. 188, Jan. 1974.

${ }^{10}$ Schroeder, J. A., Tischler, M. B., Watson, D. C., and Eshow, M. M., "Identification and Simulation Evaluation of an AH-64 Helicopter Hover Math Model," AIAA Paper 912877, August 1991.

${ }^{11}$ Cooper, G. E., and Harper, R. P., "The Use of Pilot Rating in the Evaluation of Aircraft Handling Qualities," NASA TN D-5153, 1969.

${ }^{12}$ Mack, C., Essentials of Statistics for Scientists and Technologists, Plenum Press, New York, New York, 1967, pp. 65-66. 